\title{
Operationalizing Context in Context-Aware Artifacts: Benefits and Pitfalls
}

\author{
Christopher Lueg \\ University of Technology Sydney, Australia
}

ueg@it.uts.edu.au

\begin{abstract}
The idea of context-aware artifacts is that computational artifacts are able to recognize the context in which they are being used so that these artifacts are able to adapt their functionality to the respective context. Most work in developing context-aware artifacts appears to be technology-driven by which we mean that often the relation of the artifacts to the underlying concepts of context remain unclear. In this paper, we look at the concept of context in context-aware artifacts from a cognition-oriented perspective and we argue for an explicit distinction between the concept of context that is operationalized and the original usage situation which we understand as a social setting that has been negotiated among peers in the first place. Acknowledging the difference suggests that developers of context-aware artifacts should pay considerable attention to the fact that the context determined by artifacts may differ from what the persons involved in the situation have negotiated. Furthermore, it suggests to critically review operationalizations of context in context-aware artifacts and their impact on how context is conceptualized.
\end{abstract}

Keywords: context-aware artifacts, context, situation, situatedness, negotiation.

\section{Introduction}

When referring to computational artifacts, context awareness means that artifacts are to some extent able to sense the context in which they are being used. The idea is that artifacts determine the actual context of their use in order to adapt their functionality accordingly.

An example for the potential benefit of context-aware artifacts is the idea of a context-aware mobile phone. Such a mobile would use context aspects, such as the user's identity, the user's location, and the user's current schedule, to determine the level of intrusiveness that would be appropriate when trying to notify the user of incoming calls. Notifications could range from ringing (quite intrusive) to buzzing or vibrating (less intrusive). The mobile even might suppress notifications of less important calls (not intrusive at all).

Context-aware artifacts are of particular interest to the human-computer interaction (HCI) community as the interaction with artifacts and their interfaces moves from

Material published as part of this journal, either on-line or in print, is copyrighted by the publisher of Informing Science. Permission to make digital or paper copy of part or all of these works for personal or classroom use is granted without fee provided that the copies are not made or distributed for profit or commercial advantage AND that copies 1) bear this notice in full and 2) give the full citation on the first page. It is permissible to abstract these works so long as credit is given. To copy in all other cases or to republish or to post on a server or to redistribute to lists requires specific permission and payment of a fee. Contact Editor@inform.nu to request redistribution permission. rather static desktops to less well structured environments. However, impacts of context-aware artifacts well exceed the highly inter-disciplinary field as the respective discussion of context may influence how context and context awareness is conceptualized in related disciplines. The discussion is of relevance to informing science as there are expectations that context-aware artifacts will enable context-specific information delivery. Software agents can be viewed as early software-based approaches to context-aware artifacts dealing with information. Examples discussed by Maes (1994), for example, include an agent for electronic mail handling and an agent for electronic news filtering. A more recent approach is an awareness information environment (Gross and Specht, 2001). Such an environment is expected to make use of context in order to "provide users with information that is related to their current context and therefore of most value for the coordination of the group activities".

Most work in developing context-aware artifacts appears to be technology-driven by which we mean that development is driven by what is technically feasible rather than by what might be helpful in a situation. The difference between these two approaches matters if we consider social aspects that cannot be sensed by currently available technology (see below for examples). As a consequence, the context determined by context-aware artifacts may differ from what persons involved in the situation experience. It seems that not enough effort is spent on clarifying potentially unexpected and unwanted implications of context-aware artifacts. Also, much work on context-aware artifacts seems to operationalize 
concepts of contexts that are rather simple compared to what is understood as context in academic disciplines specialized on the subject matter.

In this paper, we attempt to contribute to the discussion of context in context-aware artifacts from a cognitionoriented point of view. We proceed as follows. First, we discuss a recent technical definition of context which is typical for a lot of research in context-aware artifacts.

Based on this definition, we outline the difference between context as a characterization -or model- of a situation and the situation itself which we understand as a social setting that has been negotiated among peers in the first place. Then, we discuss implications of this distinction. Finally, we draw our conclusions and outline future research directions.

\section{Operationalizing Context in Context-Aware Artifacts}

In the anchor article of the 2001 Human Computer Interaction $(H C I)$ special issue on context-aware artifacts, Dey et al. (2001) review several definitions of context. They start with the definition given in Webster's Dictionary: "the whole situation, background or environment relevant to some happening or personality" and argue that this definition it is too general to be useful in context-aware computing. Dey et al. finally provide their own definition of context that is based on information that characterize a situation and that are relevant to the interaction between a user and its application:

\begin{abstract}
"Any information that can be used to characterize the situation of an entity, where an entity is a person, place, or object that is considered relevant to the interaction between a user and its application, including the user and the application themselves. Context is typically the location, identity and state of people, groups and computational and physical objects." (Dey et al., 2001)
\end{abstract}

This definition, as well as most other definitions of context to be found in the technical literature, indicates that context is viewed as being related to situations but the nature of this relation remains unclear. "Situation" seems to comprise "everything" while "context" consists of specific aspects that characterize a situation.

Operationalizing context by focusing on specific aspects (that characterize a situation) appears to be typical for work on context-aware artifacts. Such a focus implies that an artifact's developer has to pre-determine that some aspects, such as "location, identity and state of people, groups and computational and physical objects" (Dey et al., 2001) or "identity, locations, companions, vital signs, air quality, and network availability" (Hull et al., 1997) are significant while other aspects are less significant. This approach has several implications of which the most important ones will be discussed below:

\section{Context and the Frame Problem in AI}

The frame problem (e.g., Pylyshyn, 1987) is one of the hard problems in classical representation-based artificial intelligence. Roughly, the frame problem is about what aspects of the world have to be included in a sufficiently detailed world model and how such a world model can be kept up-to-date when the world changes. However, the world is constantly changing, intrinsically unpredictable, and infinitely rich (Pfeifer and Rademakers, 1991) and the frame problem has shown to be intractable at least in the general case (e.g., Dreyfus, 2001). Lessons to be learned from investigations of the frame problem suggest that there is little hope that research on context-aware artifacts will succeed in overcoming the problem that context understood as a model of a situation- is always limited. The frame problem is indirectly addressed by Greenberg's (2001) contribution to the $H C I$ special issue. Greenberg notes that for context-aware artifacts it may be difficult or impossible to determine an appropriate set of canonical contextual states. Also, it may be difficult to determine what information is necessary to infer a contextual state.

Of course, the hardness of the frame problem does not suggest to abandon research on context-aware artifacts but to keep in mind that such artifacts may well fail when trying to recognize a situation.

\section{Context and Situation}

Research in situatedness (e.g., Suchman, 1987; Clancey, 1997) explores the specific characteristics of usage situations. Contrary to most researchers developing context-aware artifacts, researchers in situatedness understand the characteristics of a situation as resources for (human) cognition and (human) action in the first place. This means that researchers in situatedness are not so much interested in isolating specific aspects but in understanding how and to what extent these aspects influence cognition and action when used as resources.

It is in particular the social connotation of the term "situated" that allows highlighting the differences between "context" as used in work on context-aware artifacts and the original "situation". A "situation" is an observerindependent and potentially unlimited resource that is inherently open to re-interpretation. "Context", to the contrary, as an expression of a certain interpretation of a situation is observer-dependent and therefore no longer open to re-interpretation: the meaning of aspects included in the context description is more or less determined. Other potentially relevant aspects may or may not be included in the context description; irrelevant aspects may or may not be included. The openness to re-interpretation matters as (individual) users may decide to assign significance to aspects of the environment that were not 
considered as significant before. Contrary context-aware artifacts are not capable of assigning significance.

\section{Situation and Negotiation}

"Learning, thinking, and knowing are relations among people engaged in activity in, with, and arising from the socially and culturally structured world" (Lave, 1991). One implication is that situations are always subject to negotiation among the persons involved in the situation (e.g., Wenger, 1998). There is little doubt that contextaware artifacts may provide benefit in a situation if the context they sense fits what participants in the situation have negotiated. However, as Agre (2001) notes in his contribution to the $\mathrm{HCI}$ special issue, context-aware artifacts may fail annoyingly as soon as a context-aware system's (wrong) choices become significant.

Agre's argument draws from the observation that people use the various features of their physical environment as resources for the social construction of a place, i.e., it is through their ongoing, concerted effort that the place opposed to space- comes into being. An artifact will be incapable of registering the most basic aspects of this socially constructed environment.

Context-aware buildings (also referred to as cooperative buildings) are a nice example for the potential benefit and pitfalls involved. Using currently available technology, a room in such a building could monitor its electronic schedule, the number of persons in the room, and the prevalence of business clothing among the persons in the room. The room could compute that the current context is a "business meeting context" and could instruct attendees' mobile phones not to disturb the meeting; business-related information could be projected onto the room's multipurpose walls.

However, being a social setting in the first place, a meeting does not only depend on the already mentioned aspects but also on what has been negotiated among participants of the meeting. This means that even if a particular situation fits the description of a "meeting context", the situation may have changed into an informal get together and vice versa. The subtle changes are hardly recognizable as commonly mentioned context aspects, such as the ones mentioned by Dey et al. (2001) (location, identity, state of people, groups and computational and physical objects) may not change at all. In a sense, the context does not change while the surrounding situation does. Examples for such situational changes are unexpected breaks or being well ahead of the schedule so that a meeting finishes earlier than expected. Once the meeting has changed its nature, it may no longer be appropriate to block calls and it may no longer be appropriate to project business-related information on walls (as it would demonstrate that the hosting company's expensive technology did not recognize the change in the meeting situation).

Robertson (2000) provides a nice example of a business situation that changes although all 'indicators' that could be sensed by artifacts do not appear to undergo recognizable changes. Conducting a workplace observation in a software company, Robertson attended weekly meetings over a period of seven months, making separate video and audio recordings of relevant meeting activities. One of the questions to answer was what designers were doing during these meetings. Robertson reports:

\begin{abstract}
"Amongst the talk, laughter and other activities, there was clearly a pattern to each meeting. Individuals reported what they had done while apart. Others would ask questions and each person's work would be discussed by the group. Then another person would report on her work. This process continued until everyone, who had worked on the project through the week, had told the others what she had done. Reporting was always followed by a period of shared designing, where the group worked together on some aspect of the design. Then, towards the end of the meeting, the work for the next week would be negotiated and allocated."
\end{abstract}

Robertson notes that from an observer's perspective it would be easy to divide the group's meeting into different stages, such as reporting, discussion, shared design, negotiations of future work, and finally allocation of work. One of the central findings of the workplace observation, however, was that the participants in the process did not describe their work with such labels: "[...] they did not bother with names for specific stages in their work, as they lived it, at all". Robertson concludes:

\section{"[...] naming the stages in the design work in this way excludes entirely the work of coordination and negotiation that made the process they represent possible in the first place. Moreover, this communicative work had been identified by the designers themselves as the work they most wanted supported."}

The most important point for this paper about contextaware artifacts is how the process was going on:
"[...] people did all these kinds of cooperative design work while sitting round a table talking together. At times they moved around the room, entered or left the room and moved various objects around; but there were no formal changes of position, no discernible interactional difficulties and certainly no upheaval when they changed from one kind of work to another. [...] Whatever they did was always accomplished by different combinations of their purposeful, embodied actions"

As Robertson notes, the latter was not a particularly original insight as it has been recognized by a variety of researchers from theoretical traditions including ethnomethodologists, practitioners of interaction analysis, computer-supported cooperative work researchers, exponents of distributed cognition, and so on. Researchers, such as Agre and Dourish, contributed related viewpoints to the HCI special issue. Still, we feel that these insights 
have not yet received the attention they deserve in the discussion of context-aware artifacts.

\section{Conclusions}

In this paper we have looked at context-aware artifacts from a perspective that is inspired by research in situated cognition and situated action. Accordingly, our perspective is heavily influenced by the notion of a "situation". Clearly, our notion of situation has some similarities to what is called "context" in those disciplines devoted to the study of context (see Goodwin and Duranti, 1992 for a broad overview).

Regardless of the specific terminology, we observe a tendency in context-aware artifacts towards reducing the complexity of context (in other words: alienating the term from its complex meaning in more theoretic disciplines) for technical purposes. This development resembles what happened to the term 'situated' before. The term 'situated' has its origins in the sociology literature in the context of the relation of knowledge, identity, and society. However, this social connotation of 'situated' is partly lost as the term has been reduced from something conceptual in form and social in content to merely 'interactive' or' located in some time and place' (Clancey, 1997).

We observe simplifications of context on three different levels. First, we feel that it is not particularly helpful if the term "context-aware artifacts" is used to denote artifacts as simple as air-conditioners and CO sensors (Bellotti and Edwards, 2000) or automatically flushing toilets (Winograd, 2000). The point of concern, however, is not the simplicity of these artifacts. Rather, it is the exclusion of the social and cognitive processes that generate context.

Second and more importantly, work on context-aware artifacts is typically based on having developers define what aspects of the world become context. This means that developers -as observers- assign significance. Often, researchers start with comprehensive definitions but operationalize much simpler concepts of context. A good example is the already discussed definition provided by Dey et al. (2001). Another example is provided by Gross and Prinz (2000) defining an (awareness) context as "the inter-related conditions in which something exists or occurs". While the definition is rather comprehensive, the actual implementation of the definition in their awareness architecture consists of a number of explicitly defined attributes, such as human members of a context, physical locations related to a context, and artifacts of a context. Such simplifications appear to be necessary when developing specific artifacts. What is missing, however, is a discussion of the difference between context in its original complex definition and the context model that is finally implemented. After all, an artifact's capability to be "context-aware" depends on this model of context.

Finally, we observe an effort in developing architectures for (managing) context (e.g., Dey et al. 2001, Winograd, 2001). Bringing software engineering principles to the development of context-aware artifacts is certainly a positive development. However, from the point of view expressed in this paper, we observe a lack of precision in terminology. As Winograd (2001) notes: "features of the world become context through their use", i.e., something is not context because of its inherent properties but because of the way it is used in interpretation. The point is that architectures for (managing) context do not manage context but models of context (or: representations of context). The difference matters because again the social processes that generate context in the first place are excluded.

\section{Future Research}

It seems that some research in context-aware artifacts is implicitly based on the assumption that context is rather static in some application domains and that it is therefore possible to represent context in rather static data structures. This is our interpretation of approaches, such as Winograd's (2001) architecture for modeling context or the awareness environment discussed by Gross and Prinz (2000). It is a particular benefit of the latter paper that modeling application contexts is explicitly mentioned as an integral activity to be performed by users of the architecture. The understanding of context also shapes future research in this area. Open questions are, for example, who is responsible for modeling contexts, how can contexts be kept up-to-date, and who is going to update contexts (Gross and Prinz, 2000). Based on the theoretic considerations presented in this paper, we are more interested in questions, such as in which situations models of context work (and why) and in which they fail (and why). These questions can only be answered empirically but unfortunately hardly any data about the usage of context-aware artifacts is available.

Furthermore, we are interested in the question whether context-aware artifacts indeed provide the benefit expected. In most cases, people are well aware of their situation and have quite some expertise in using artifacts in an appropriate way (e.g., most people turn off their mobiles during a theater performance because they know that mobiles ringing during theater audiences are annoying). People are also good at recognizing situation changes as they are part of the negotiations that lead to changes. In other words, what is the benefit of making artifacts context-aware over making artifacts easier to use? 


\section{References}

Agre, P. E. (2001). Changing places: contexts of awareness in computing. Human-Computer Interaction, 16(2-3).

Bellotti, V. and Edwards, K. (2001). Intelligibility and accountability: human considerations in context aware systems. Human-Computer Interaction, 16(2-3).

Clancey, W. J. (1997). Situated cognition. Cambridge University Press.

Dey, A. K., Salber, D., and Abowd, G. D. (2001). A conceptual framework and a toolkit for supporting the rapid prototyping of context-aware applications. Human-Computer Interaction, 16(2-3).

Dreyfus, H. (2001). On the Internet. Routledge, London, UK.

Goodwin, C. and Duranti, A. (1992). Rethinking context: an introduction. In Duranti, A. and Goodwin C. Rethinking context: language as an interactive phenomenon. Cambridge University Press.

Greenberg, S. (2001). Context as dynamic construct. HumanComputer Interaction, 16(2-3).

Gross, T. and Specht M. (2001) Awareness in context-aware information systems. Tagungsband der 1. Fachübergreifenden Konferenz "Mensch \& Computer", Bonn, Germany.

Gross, T. and Prinz, W. (2000). Gruppenwahrnehmung im Kontext. Tagungsband der Deutschen Computer-Supported Cooperative Work Konferenz (D-CSCW), Munich, Germany, pages 115126. Teubner, Stuttgart, Germany.

Hull, R., Neaves, P., and Bedford-Roberts, J. (1997). Towards situated computing. In Proceedings of the First International Symposium on Wearable Computers (ISWC'97), pages 146153, IEEE.

Lave, J. (1991). Situated learning in communities of practice. In Resnick, L. B., Levine, J. M., and Teasley, S. D., editors, Perspectives on Socially Shared Cognition, pages 63-82.
American Psychological Association, Washington, DC, USA. Third Printing April 1996.

Maes, P. (1994). Agents that reduce work and information overload. Communications of the ACM, 37(7), pages 31-40.

Pfeifer, R. and Rademakers, P. (1991). Situated Adaptive Design: Toward a Methodology for Knowledge Systems Development. In Brauer, W. and Hernandez, D., editors, Proceedings of the Conference on Distributed Artificial Intelligence and Cooperative Work, pages 53-64. Springer-Verlag.

Pylyshyn, Z. W., editor (1987). The robot's dilemma: the frame problem in artificial intelligence. Ablex Publishing Corporation, Norwood, NJ.

Robertson, T. (2000). Building bridges: negotiating the gap between work practice and technology design. Human-Computer Studies, 53, 121-146.

Suchman, L. A. (1987). Plans and Situated Actions - The Problem of Human-Machine Communication. Cambridge University Press.

Wenger, E. (1998). Communities of practice: learning, meaning, and identity. Cambridge University Press. First Paperback Edition 1999.

Winograd, T. (2001). Architectures for context. Human-Computer Interaction, 16(2-3).

\section{Biography}

Dr. Christopher Lueg is a Senior Lecturer in Information Systems at the Faculty of Information Technology, University of Technology Sydney (Australia). He has a diploma in Computer Science from the University of Dortmund (Germany) and a doctoral degree from the Faculty of Science, University of Zurich (Switzerland). His interests are trans-disciplinary in the intersection of computer science, information science, and cognitive science. 